\title{
Fully electromagnetic nonlinear gyrokinetic equations for tokamak edge turbulence
}

\author{
Hahm, T.S.; Wang, Lu; Madsen, Jens
}

Published in:

Physics of Plasmas

Link to article, DOI:

$10.1063 / 1.3073671$

Publication date:

2009

Document Version

Publisher's PDF, also known as Version of record

Link back to DTU Orbit

Citation (APA):

Hahm, T. S., Wang, L., \& Madsen, J. (2009). Fully electromagnetic nonlinear gyrokinetic equations for tokamak edge turbulence. Physics of Plasmas, 16(2), 022305. https://doi.org/10.1063/1.3073671

\section{General rights}

Copyright and moral rights for the publications made accessible in the public portal are retained by the authors and/or other copyright owners and it is a condition of accessing publications that users recognise and abide by the legal requirements associated with these rights.

- Users may download and print one copy of any publication from the public portal for the purpose of private study or research.

- You may not further distribute the material or use it for any profit-making activity or commercial gain

- You may freely distribute the URL identifying the publication in the public portal

If you believe that this document breaches copyright please contact us providing details, and we will remove access to the work immediately and investigate your claim 


\title{
Fully electromagnetic nonlinear gyrokinetic equations for tokamak edge turbulence
}

\author{
T. S. Hahm, ${ }^{1}$ Lu Wang, ${ }^{1, a)}$ and J. Madsen ${ }^{2}$ \\ ${ }^{1}$ Princeton Plasma Physics Laboratory, Princeton University, P.O. Box 451, Princeton, \\ New Jersey 08543, USA \\ ${ }^{2}$ Association EURATOM-Ris $\phi$ DTU, Ris $\phi$ National Laboratory for Sustainable Energy, \\ Technical University of Denmark, P.O. 49, DK-4000 Roskilde, Denmark
}

(Received 21 August 2008; accepted 26 December 2008; published online 13 February 2009)

\begin{abstract}
An energy conserving set of the fully electromagnetic nonlinear gyrokinetic Vlasov equation and Maxwell's equations, which is applicable to both L-mode turbulence with large amplitude and H-mode turbulence in the presence of high $\mathbf{E} \times \mathbf{B}$ shear has been derived. The phase-space action variational Lie perturbation method ensures the preservation of the conservation laws of the underlying Vlasov-Maxwell system. Generalized ordering takes $\rho_{i} \ll \rho_{\theta i} \sim L_{E} \sim L_{p} \ll R$ [here $\rho_{i}$ is the thermal ion Larmor radius and $\left.\rho_{\theta i}=B /\left(B_{\theta} \rho_{i}\right)\right]$, as typically observed in the tokamak H-mode edge, with $L_{E}$ and $L_{p}$ being the radial electric field and pressure gradient lengths. $k_{\perp} \rho_{i} \sim 1$ is assumed for generality, and the relative fluctuation amplitudes $e \delta \phi / T_{i} \sim \delta B / B$ are kept up to the second order. Extending the electrostatic theory in the presence of high $\mathbf{E} \times \mathbf{B}$ shear [Hahm, Phys. Plasmas 3, 4658 (1996)], contributions of electromagnetic fluctuations to the particle charge density and current are explicitly evaluated via pullback transformation from the gyrocenter distribution function in the gyrokinetic Maxwell's equation. (C) 2009 American Institute of Physics.
\end{abstract}

[DOI: $10.1063 / 1.3073671]$

\section{INTRODUCTION}

Understanding tokamak microturbulence is required for developing a predictive capability of tokamak transport. The nonlinear gyrokinetic formulations ${ }^{1-7}$ have provided theoretical foundations for recent advances in nonlinear gyrokinetic simulations of tokamak microturbulence. Both formulations and simulations have traditionally focused on tokamak core turbulence in which the fluctuation amplitude is relatively small, i.e., less than or comparable to $\delta n / n_{0} \sim 10^{-2}$ and the gradients in macroscopic parameters, such as, pressure are relatively mild. ${ }^{8}$

As experience in enhancing tokamak confinement ${ }^{9-13}$ accumulates, for instance, by H-mode operation, tokamak edge plasmas became hotter and less collisional. Therefore, traditional edge turbulence simulations ${ }^{14-16}$ based on Braginskiitype fluid equations are not strictly applicable in some of the leading tokamaks in the present and future. For more accurate simulations of such collisionless edge plasmas, kinetic effects associated with long mean free path and finite orbit sizes should be included properly. However, the following obvious challenges must be faced when one tries to apply the existing nonlinear gyrokinetic formalism derived mainly for core turbulence:

i) Fluctuation amplitudes in L-mode edge plasmas are typically on the order of $\delta n / n_{0} \sim 10^{-1}$ inside the last closed flux surface (LCFS), ${ }^{17,18}$ and can be even higher in the scrape off layer (SOL). ${ }^{19-22}$

ii) After an H-mode transition, $E_{r}$ well is formed just inside the LCFS. The pressure gradient scale length and the radial electric field scale length in the edge $E_{r}$ well is on the

\footnotetext{
${ }^{a}$ Also at: Department of Physics, Peking University, Beijing 100871, China.
}

order of the ion poloidal gyroradius, ${ }^{23} \rho_{\theta i} \equiv v_{T i} M c /\left(e B_{\theta}\right)$.

iii) Not only in $\mathrm{H}$-mode plasmas, but also in some L-mode plasmas, we have $\rho_{i} / L_{p}>L_{p} / R_{0}$ at the edge. Since $\rho_{i}$ is a typical unit for the radial scale of microturbulence, and $L_{p}$ is a length scale of a macroscopic quantity, we have an intriguing situation where one of the primary smallness parameters $\rho_{i} / L_{p}$ in the conventional nonlinear gyrokinetic formulation $^{1}$ is greater than a ratio between two macroscopic scale lengths.

In retrospect, many theoretically related issues have been addressed in the context of nonlinear gyrokinetic equations for core transport barriers. ${ }^{24}$ That work, however, only considered the electrostatic fluctuations. The present work provides an extension of the electrostatic nonlinear gyrokinetic equations as presented in Ref. 24 to fully electromagnetic edge turbulence in toroidal geometry, with an ordering applicable to both large fluctuation amplitudes in L-mode and residual fluctuations in the presence of strong $\mathbf{E} \times \mathbf{B}$ in $\mathrm{H}$-mode. It has been shown that the edge turbulence is electromagnetic even for low local values of plasma $\beta .^{14,25}$ As emphasized in the context of both theory ${ }^{26-32}$ and experiments, ${ }^{10-13} \mathbf{E} \times \mathbf{B}$ flow shear rather than plasma mass flow shear of a particular species plays a more fundamental role in reducing turbulence. It is also natural to deal with the electromagnetic fields, $\mathbf{E}$ and $\mathbf{B}$, rather than plasma flows, in formulating the nonlinear gyrokinetic equations which are based on the equations of motion for a single charged particle's gyrocenter.

The emphasis in Ref. 24 was on a systematic description of the collective phenomena (i.e., a self-consistent treatment of turbulence) in which the Vlasov equation and the Maxwell's equation are treated on an equal footing with various 
TABLE I. Progress in nonlinear gyrokinetic formulations in a torus with flows via Lie perturbation method. Vacant slots indicate that the specific item has not been performed in that particular paper. Explicit evaluation here means performing the integration over velocity space following the pullback transformation, for quantities such as polarization density and magnetization terms. ES and EM stand for electrostatic and electromagnetic fluctuations, respectively.

\begin{tabular}{|c|c|c|c|c|}
\hline & Brizard ${ }^{\prime} 95^{\mathrm{a}}$ & Hahm $96^{\text {b }}$ & $\begin{array}{l}\text { Qin et al. }{ }^{\prime} 06,{ }^{\mathrm{c}},{ }^{\prime} 07^{\mathrm{d}} \\
\text { Kawamura et al., }, 08^{\mathrm{e}}\end{array}$ & This work \\
\hline $\begin{array}{l}\text { Characteristics of } \\
\text { fluctuations appearing } \\
\text { in the GK Vlasov equation }\end{array}$ & EM & ES & EM & EM \\
\hline $\begin{array}{l}\text { Explicit evaluation of } \\
\text { GK Poisson's equation }\end{array}$ & & for ES & for ES & for EM \\
\hline $\begin{array}{l}\text { Explicit evaluation of } \\
\text { GK Ampere's law }\end{array}$ & & & & for EM \\
\hline $\begin{array}{l}\text { Expression for total } \\
\text { energy invariant }\end{array}$ & & yes & & yes \\
\hline $\begin{array}{l}\text { Reference } 48 . \\
{ }^{\mathrm{a}} \text { Reference } 24 . \\
{ }^{\mathrm{c}} \text { Reference } 33 . \\
{ }^{\mathrm{d}} \text { Reference } 34 . \\
{ }^{\mathrm{e}} \text { Reference } 35 .\end{array}$ & & & & \\
\hline
\end{tabular}

terms explicitly derived from the general expressions, and with an explicit expression for the total energy invariant which can be used as an indicator of accuracy in numerical simulations. This underlying philosophy followed those of the early modern nonlinear gyrokinetic theories ${ }^{3-6}$ and a recent review. ${ }^{7}$ It is noteworthy that the necessity of extending nonlinear gyrokinetic formulations to edge turbulence in the presence of flow shear has been widely recognized. Recent publications based on the Lie perturbation theory including Refs. 33-35 used the $\mathbf{E} \times \mathbf{B}$ flow, rather than the ion mass flow, following the approach in Ref. 24. On the other hand, the emphasis of Refs. 33 and 34 was mostly on a systematic manifestation of modern nonlinear gyrokinetic theory in the context of the language of differential geometry. Utilizing noncanonical Hamiltonian theories of guiding center drifts, including that by Littlejonhn, ${ }^{36-38}$ more drift terms, including the polarization drift associated with a time-dependent background radial electric field, are kept in the gyrokineticVlasov equation. Reference 35 has shown that by adopting a modified definition of the $\mathbf{E} \times \mathbf{B}$ flow, higher accuracy in the guiding center drift can be achieved over the formulations in Ref. 33. However, in those recent publications, ${ }^{33-35}$ the explicit form of the gyrokinetic Ampere's law after the integrations over velocity space for the electrical current has not been derived. Furthermore, the total energy invariant extending that in Ref. 24 has not been derived to date for the electromagnetic case. The progress in nonlinear gyrokinetic formulations with flows in tokamak geometry via the Lie perturbation method is summarized in Table I.

In this paper, an energy-conserving and phase-space volume conserving set of the fully electromagnetic nonlinear gyrokinetic Vlasov equation and Maxwell's equation, which is applicable to tokamak edge turbulence, is derived. These conservation properties become more important as long term gyrokinetic simulations well beyond the nonlinear saturation phase are being pursued with recent advances in computational power. ${ }^{39}$

The principal results of this paper are as follows:

i) An energy-conserving set of the fully electromagnetic nonlinear gyrokinetic Vlasov and Maxwell's equations is derived for the first time in the presence of strong $\mathbf{E} \times \mathbf{B}$ flow shear. Symplectic derivation via phase-space Lagrangian Lie-perturbation theory ensures the preservation of the conservation laws.

ii) Expressions for the gyrokinetic Maxwell equations alongside corresponding energy invariants are presented for practically useful limiting cases, i.e., the long wavelength limit and for a Maxwellian distribution in $\mu$.

iii) In particular, various contributions of both shearAlfvénic $\left(\delta A_{\|}\right)$and compressional $\left(\delta B_{\|}\right)$electromagnetic fluctuations to the gyrokinetic Poisson equation and the gyrokinetic Ampere's law are explicitly evaluated in the presence of strong $\mathbf{E} \times \mathbf{B}$ flow shear.

The remainder of this paper is organized as follows. In Sec. II, the guiding-center motion in the presence of strong $\mathbf{E}$ $\times \mathbf{B}$ shear is presented. The gyrophase-independent EulerLagrange equation for the gyrocenter drift in the presence of the electromagnetic fluctuation is derived in Sec. III. In Sec. IV, an energy conserving set of general gyrokinetic VlasovMaxwell equations with strong $\mathbf{E} \times \mathbf{B}$ shear is derived. Their limiting forms for long wavelength fluctuations and for a Maxwellian distribution in $\mu$ are also presented, respectively. Major emphasis is placed on the rigorous and transparent derivation of the most general result via the phase-space Lagrangian Lie-perturbation theory, and the explicit evaluation of velocity moments involving the gyrocenter distribution function which appear in the gyrokinetic Maxwell equations. 


\section{GUIDING-CENTER DRIFT IN THE PRESENCE OF STRONG EXB FLOW SHEAR}

In this section, we present the guiding-center equations of motion in the presence of strong radial electric field shear and steep pressure gradients as observed in most H-mode edges. As emphasized in the previous work on the nonlinear gyrokinetic equations in core transport barriers, ${ }^{24}$ a formulation in terms of the radial electric field rather than in terms of mass flow is preferred. Since the single particle's guiding center motion is determined by the electromagnetic field rather than the mass flow, this choice is not only natural, but also advantageous in separating the issue of determining the equilibrium ion distribution function (which is also an important issue at the tokamak edge in its own right ${ }^{40,41}$ ) from the formulation of the nonlinear gyrokinetic equation for turbulence. Neoclassical equilibrium, i.e., the distribution function in the absence of the turbulence, in the steep pressure gradient edge region, can be calculated numerically as an input for turbulence simulations. ${ }^{42-45}$ A massively parallel Monte Carlo guiding center simulation could tabulate the distribution function in the 4D phase space. We focus only on the issues involving turbulence in this paper without specifying the equilibrium mass flow. This approach is thus conceptually simpler than previous nonlinear gyrokinetic formulations in terms of the relative velocity in the frame moving with the mass flow. ${ }^{46-49}$

The standard nonlinear gyrokinetic ordering ${ }^{1}$ consists of

$$
\omega / \Omega_{i} \sim \rho_{i} k_{\|} \sim \delta B / B \sim e \delta \phi / T_{i} \sim \epsilon
$$

and

$$
k_{\perp} \rho_{i} \sim 1,
$$

where $\omega$ and $\Omega$ are the characteristic fluctuation frequency and the ion cyclotron frequency, respectively; $k_{\|}$and $k_{\perp}$ are the components of the wave vector in the parallel and perpendicular direction with respect to the magnetic field; $\rho_{i}$ $=\left(T_{i} / M\right)^{1 / 2} / \Omega_{i}$ is the thermal ion gyroradius; $\delta \phi$ is the fluctuating electrostatic potential; $\delta B$ is the fluctuating magnetic field; and $\epsilon \ll 1$ is a small ordering parameter. A tokamakspecific ordering, $B_{\theta} / B \simeq r q / R \ll 1$, will be used in this work to differentiate the poloidal gyroradius from the gyroradius. Here, $r / R$ is the local inverse aspect ratio, and $q$ is the magnetic safety factor.

For a derivation of the unperturbed phase-space Lagrangian of a charged particle which is relevant for edge plasma conditions including the $\mathrm{H}$-mode state, it is useful to summarize the following key experimental findings. A spontaneous $\mathrm{H}$-mode transition ${ }^{9}$ starts with a rapid increase in the negative radial electric field $E_{r}$ at the edge just inside the LCFS. Well after the transition, a negative $E_{r}$ well is formed and the steep ion pressure gradient in the same region becomes large. Quite often, it becomes the dominant contributor to $E_{r}$ in the radial force balance relation for the main ion species, ${ }^{23,50,51}$ i.e., $E_{r} \sim 1 /\left(n_{i} e_{i}\right) \partial P_{i} / \partial r$. For these plasmas, the gradient lengths of $E_{r}$ and $P_{i}$ are of the same order as the ion poloidal gyroradius, i.e., $L_{E} \sim L_{p} \sim \rho_{\theta i}$. These conditions correspond to $u_{E}^{(0)} \equiv-c E_{r} / B \sim u_{* i} \equiv-\left(c / n_{i} e_{i} B\right) \partial P_{i} / \partial r$ $\sim\left(\rho_{i} / L_{p}\right) v_{T i}$, and $e \Phi^{(0)} / T_{i} \sim 1$. While $\rho_{\theta i} / L_{p} \sim 1$, we can still identify a small parameter for the unperturbed particle orbit calculations in tokamak plasmas with large $E_{r}$ shear, except for low aspect ratio experiments, such as, the National Spherical Torus eXperiment (NSTX), ${ }^{52}$

$$
\epsilon_{E} \equiv \frac{\rho_{i}}{L_{E}} \sim \frac{\rho_{i}}{L_{p}} \sim \frac{B_{\theta}}{B} \ll 1
$$

We note that $\epsilon_{E}$ is larger than the conventional small parameter $\delta_{B}$ for the unperturbed particle orbit calculations in the absence of large $E_{r}$ shear,

$$
\delta_{B} \equiv \frac{\rho_{i}}{L_{B}} \ll 1,
$$

where $L_{B}^{-1} \equiv|\partial B / B \partial r|$ originates from the inhomogeneity in the equilibrium $\mathbf{B}$ field.

We will discuss the relation between $\epsilon_{E}$ and $\delta_{B}$ for our problem shortly. Starting from the unperturbed phase-space Lagrangian of a charged particle, one can perform Lie perturbation analysis as described in Refs. 6, 7, 24, 38, and 48, to obtain the guiding-center phase-space Lagrangian,

$$
\gamma_{0} \equiv\left(\frac{e}{c} \mathbf{A}+M \mathbf{u}_{E}+p_{\|} \mathbf{b}\right) \cdot d \mathbf{R}+\frac{\mu B}{\Omega} d \theta-H_{0} d t .
$$

Here the notations follow mostly those used in Ref. 24. The noncanonical guiding-center coordinates which simplify the phase-space Lagrangian are used. $\mathbf{R} \equiv \mathbf{x}-\boldsymbol{\rho}, \mu$ is the guidingcenter magnetic moment in the frame moving with $\mathbf{u}_{E} \equiv c \mathbf{b}$ $\times \nabla \Phi / B,{ }^{36}$ associated with the equilibrium potential $\Phi . p_{\|}$is the guiding center parallel kinetic momentum which includes the Banos drift, ${ }^{53}$ and $\theta$ is the gyrophase angle. More detailed discussions on the choice of guiding center variables can be found in Ref. 6. On the right-hand side of Eq. (3), the $O\left(\delta_{B}\right)$ term $-\mu B / \Omega\left[\nabla \mathbf{e}_{1} \cdot \mathbf{e}_{2}+\frac{1}{2}(\mathbf{b} \cdot \nabla \times \mathbf{b}) \mathbf{b}\right] \cdot d \mathbf{R}$ is ignored for simplicity. The term $\mu \nabla \mathbf{e}_{1} \cdot \mathbf{e}_{2}$ which depends on the choice of perpendicular unit vectors $\mathbf{e}_{1}$ and $\mathbf{e}_{2}$, is related to a gyrogauge invariance. ${ }^{38}$ In Eq. (3), the guiding-center Hamiltonian up to the order $\epsilon_{E}^{2}$ is

$$
H_{0}=e \Phi+\mu B+\frac{p_{\|}^{2}}{2 M}+\frac{M}{2} u_{E}^{2}+\frac{\mu B}{2 \Omega} \mathbf{b} \cdot \nabla \times \mathbf{u}_{E},
$$

and $\mu \mathbf{b} \cdot \nabla \times \mathbf{u}_{E} \simeq(c \mu / B) \nabla_{\perp}^{2} \Phi$ describes the finite Larmororbit-average reduction of the equilibrium potential. ${ }^{48} \mathrm{We}$ note that unlike typical core profiles, the tokamak edge profiles satisfy $\rho_{i} / L_{p}>L_{p} / R$. Therefore, we assume $\epsilon_{E}^{2}>\delta_{B}$.

In passing, we remark that the trapped ion radial width modification due to $E_{r}$ shear ${ }^{54-57}$ is on the order of unity for our ordering based on typical tokamak H-mode edge plasma parameters. This can be easily shown from the fact that in general toroidal geometry, the banana orbit modification parameter $^{58}$ is given by

$$
S \equiv 1+\frac{M c^{2}}{e} \frac{\left(R B_{\phi}\right)^{2}}{\left\langle B^{2}\right\rangle} \frac{\partial}{\partial \psi}\left(\frac{E_{r}}{R B_{\theta}}\right) .
$$

On the other hand, the $\mathbf{E} \times \mathbf{B}$ shearing rate in general toroidal geometry ${ }^{29,31}$ is given by 


$$
\omega_{E}=\frac{\Delta r_{0}}{\Delta l_{\perp}} \frac{c\left(R B_{\theta}\right)^{2}}{B} \frac{\partial}{\partial \psi}\left(\frac{E_{r}}{R B_{\theta}}\right) .
$$

Here, $\psi$ is the poloidal flux representing the radial coordinate via $d \psi=R B_{\theta} d r, \Delta r_{0}=\Delta \psi_{0} / R B_{\theta}$ is the radial correlation length, and $\Delta l_{\perp}=R B_{\theta} \Delta \phi / B$ is the correlation length of the ambient turbulence in the direction perpendicular to the field line, but within the flux surface. Therefore, for near isotropic ambient turbulence, $\Delta r_{0} \simeq \Delta l_{\perp}$, they are related through ${ }^{59}$

$$
S \simeq 1+\left(\frac{B}{B_{\theta}}\right)^{2} \frac{\omega_{E}}{\Omega_{i}} .
$$

Since $\omega_{E} / \Omega_{i} \sim \epsilon_{E}^{2}$ from Eq. (1), we have $|S-1| \sim 1$, and we have an order of unity banana orbit width modification due to $\mathbf{E} \times \mathbf{B}$ shear. It is obvious that the appearance of the particular combination $E_{r} / R B_{\theta}$ in both the $\mathbf{E} \times \mathbf{B}$ shearing rate and the orbit modification factor is a consequence of the axisymmetry in tokamak geometry. Based on our ordering, the ion gyro-orbit is near circular in the frame moving with the equilibrium $\mathbf{E} \times \mathbf{B}$ velocity.

In general, the variation of the fundamental one-form, $\gamma \equiv \gamma_{\mu} d z^{\mu}=\gamma_{i} d z^{i}-h d t$, yields the Euler-Lagrange equation ${ }^{37}$

$$
\left(\frac{\partial \gamma_{j}}{\partial z^{i}}-\frac{\partial \gamma_{i}}{\partial z^{j}}\right) \frac{d z^{j}}{d t}=\frac{\partial h}{\partial z^{i}}+\frac{\partial \gamma_{i}}{\partial t} \text {. }
$$

For the unperturbed phase-space Lagrangian given by Eq. (3), the nontrivial components of Eq. (5) are

$$
\begin{aligned}
-\frac{e}{c} \mathbf{B}^{*} \times \frac{d \mathbf{R}}{d t}-\mathbf{b} \frac{d p_{\|}}{d t}= & \nabla\left[e \Phi+\mu B+p_{\|}^{2} /(2 M)\right. \\
& \left.+(M / 2) u_{E}^{2}+\frac{\mu B}{2 \Omega} \mathbf{b} \cdot \nabla \times \mathbf{u}_{E}\right] .
\end{aligned}
$$

Following the same decomposition procedure described in Ref. 4, one obtains the following gyrocenter equations of motion:

$$
\begin{aligned}
\frac{d \mathbf{R}}{d t}= & \frac{p_{\|}}{M} \frac{\mathbf{B}^{*}}{B_{\|}^{*}}+\frac{c \mathbf{b}}{e B_{\|}^{*}} \times[e \nabla \Phi+\mu \nabla B \\
& \left.+\frac{\mu B}{2 \Omega} \nabla\left(\mathbf{b} \cdot \nabla \times \mathbf{u}_{E}\right)+\frac{M}{2} \nabla\left(u_{E}^{2}\right)\right],
\end{aligned}
$$

and

$$
\begin{aligned}
\frac{d p_{\|}}{d t}= & -\frac{\mathbf{B}^{*}}{B_{\|}^{*}} \cdot\left[e \nabla \Phi+\mu \nabla B+\frac{\mu B}{2 \Omega} \nabla\left(\mathbf{b} \cdot \nabla \times \mathbf{u}_{E}\right)\right. \\
& \left.+\frac{M}{2} \nabla\left(u_{E}^{2}\right)\right] .
\end{aligned}
$$

Here

$$
\mathbf{B}^{*} \equiv \mathbf{B}+\frac{M c}{e} \nabla \times\left(\mathbf{u}_{E}+\frac{p_{\|}}{M} \mathbf{b}\right),
$$

and

$$
B_{\|}^{*} \equiv \mathbf{b} \cdot \mathbf{B}^{*}=B\left\{1+\frac{\mathbf{b}}{\Omega} \cdot \nabla \times\left(\mathbf{u}_{E}+\frac{P_{\|}}{M} \mathbf{b}\right)\right\} .
$$

\section{LIE-PERTURBATION ANALYSIS WITH FULLY ELECTROMAGNETIC FLUCTUATIONS}

In this section, we introduce the time-dependent electromagnetic fluctuations corresponding to tokamak edge turbulence. It has been almost universally observed ${ }^{17,18,60}$ that the relative density fluctuation amplitude $\delta n / n_{0}$ increases from the core to the edge monotonically in tokamak plasmas when there are no transport barriers. In the core, the level is often less than $1 \%,{ }^{8,11,13}$ while towards the LCFS, it typically reaches $\sim 10^{-1}$. In the SOL, it can be sometimes as high as 0.25 in the form of long-lived, spatially intermittent blobs. ${ }^{22,61}$ In this paper, we pursue the nonlinear gyrokinetic approach with full ion Larmor radius effects $k_{\perp} \rho_{i} \sim 1$, and strong turbulence,

$$
\epsilon_{\phi} \equiv \frac{\delta f}{f_{0}} \sim \frac{e \delta \phi}{T_{i}} \sim \frac{\delta B}{B_{0}} \ll 1 .
$$

While the relative magnetic fluctuation level for edge microturbulence, in the absence of low-mode-number MHD activity, is measured to be much smaller than that of the electrostatic fluctuations, ${ }^{20}$ we order them to be comparable for generality. We perform the perturbation theory derivation up to the second order in the relative fluctuation amplitude, i.e., in $\epsilon_{\phi}$. By taking the nonlinear mode coupling term comparable to the linear driving term from the equilibrium pressure gradient, we get the mixing length type balance, ${ }^{62,63}$ $\delta f / f_{0} \sim 1 / k_{\perp} L_{p}$ which is equivalent to taking $\epsilon_{\phi} \sim \epsilon_{E}$ for $k_{\perp} \rho_{i} \sim 1$. Note that we maintain consistency in ordering by keeping terms up to the second order both in $\epsilon_{\phi}$ and in $\epsilon_{E}$ $=\rho_{i} / L_{E}$.

While one could also pursue a drift-kinetic type ordering of $e \delta \phi / T_{i} \sim 1$ and $k_{\perp} \rho_{i} \ll 1$, we believe that it is important to treat the relatively short wavelength fluctuations at $k_{\perp} \rho_{i} \sim 1$, which nonlinearly interact with the longer wavelength fluctuations accurately, even though their amplitudes at saturation are small. ${ }^{64}$ The necessity of resolving edge turbulence down to the scale $k_{\perp} \rho_{i} \sim 1$ has been demonstrated in Ref. 25 . It is also encouraging to note that there seems to be a growing recognition ${ }^{65}$ that the final form of the nonlinear gyrokinetic equation is robust in the drift kinetic regime. A related explicit illustration exists ${ }^{66}$ for the electromagnetic nonlinear gyrokinetic equations in a simple geometry ${ }^{5}$ in detail.

Fluctuations are introduced in the first order phase space Lagrangian in terms of the four-potential $(\delta \phi, \delta \mathbf{A})$,

$$
\begin{aligned}
\gamma_{1}= & \frac{e}{c} \delta \mathbf{A}(\mathbf{R}+\rho, t) \cdot(d \mathbf{R}+d \rho)-e \delta \phi(\mathbf{R}+\rho, t) d t \\
= & \frac{e}{c}\left(\delta \mathbf{A}_{g c} \cdot d \mathbf{R}+\delta \mathbf{A}_{g c} \cdot \frac{\partial \rho}{\partial \mu} d \mu+\delta \mathbf{A}_{g c} \cdot \frac{\partial \rho}{\partial \theta} d \theta\right) \\
& -e \delta \phi_{g c} d t .
\end{aligned}
$$

Then, the Lie-perturbation analysis consists of finding near-identity transformations, order by order, which eliminate the gyrophase dependence in Eq. (9) introduced by the fact that the fluctuating electromagnetic potentials are functions of the particle position $\mathbf{x} \equiv \mathbf{R}+\boldsymbol{\rho}$, rather than functions of the guiding center position $\mathbf{R}$, 


$$
\begin{aligned}
& \Gamma_{0}=\gamma_{0}+d S_{0}, \\
& \Gamma_{1}=\gamma_{1}-L_{1} \gamma_{0}+d S_{1} .
\end{aligned}
$$

Here, $\gamma_{0}$ is given by Eq. (3),

$$
\left(L_{1} \gamma\right)_{\mu}=g_{1}^{\nu}\left(\frac{\partial \gamma_{\mu}}{\partial z^{\nu}}-\frac{\partial \gamma_{\nu}}{\partial z^{\mu}}\right)
$$

where $g_{1}^{\nu}$ is the generator of the Lie transformation. With $d S_{0}=0$ and $g_{1}^{t}=0$, Eq. (11) yields

$$
\begin{aligned}
& \Gamma_{1}=d S_{1}+\frac{e}{c}\left(\delta \mathbf{A}_{g c} \cdot d \mathbf{R}+\delta \mathbf{A}_{g c} \cdot \frac{\partial \rho}{\partial \mu} d \mu+\delta \mathbf{A}_{g c} \cdot \frac{\partial \rho}{\partial \theta} d \theta\right) \\
& -e \delta \phi_{g c} d t+\frac{M c}{e} g_{1}^{\theta} d \mu-\frac{M c}{e} g_{1}^{\mu} d \theta+\mathbf{b} \cdot \mathbf{g}_{1}^{\mathbf{R}} d p_{\|}
\end{aligned}
$$

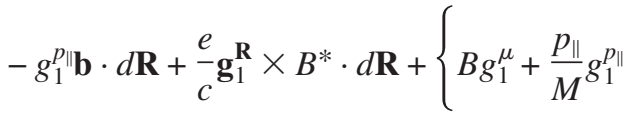

$$
\begin{aligned}
& +\mathbf{g}_{1}^{\mathbf{R}} \cdot\left[e \nabla \Phi+\mu \nabla B+\frac{M}{2} \nabla\left(u_{E}^{2}\right)\right. \\
& \left.\left.+\frac{\mu B}{2 \Omega} \nabla\left(\mathbf{b} \cdot \nabla \times \mathbf{u}_{E}\right)\right]\right\} d t
\end{aligned}
$$

where

$$
\mathbf{g}_{1}^{\mathbf{R}} \equiv\left(g_{1}^{\mathbf{R}_{1}}, g_{1}^{\mathbf{R}_{2}}, g_{1}^{\mathbf{R}_{3}}\right) .
$$

In Eq. (13), one can choose $d S_{1}$ and $g_{1}^{\nu}$ such that all of the $\Gamma_{1 \nu}$ vanish except for $\Gamma_{1 t}$. This choice corresponds to the Hamiltonian approach (rather than the symplectic approach) adopted in Ref. 5. Also, by requiring that there be no $\theta$-dependent term in $\Gamma_{1 t}$, one obtains

$$
\begin{aligned}
\Gamma_{1 t} \equiv-e\left\langle\delta \psi_{g c}\right\rangle= & -e\left[\left\langle\delta \phi_{g c}\right\rangle-\frac{1}{c}\left(\frac{\bar{p}_{\|}}{M} \mathbf{b}+\mathbf{u}_{E}\right) \cdot\left\langle\delta \mathbf{A}_{g c}\right\rangle\right. \\
& \left.-\frac{1}{c}\left\langle\mathbf{c}_{\perp} \cdot \delta \mathbf{A}_{g c}\right\rangle\right] .
\end{aligned}
$$

Here, the contribution from $\mathbf{v}_{d}$ is neglected, because $\delta_{B}$ $\ll \epsilon_{E}$. It is important to note that $\bar{p}_{\|} \simeq p_{\|}+e / c \delta A_{\| g c}$, which is close to the canonical momentum including the $\delta A_{\|}$ contribution. The bracket denotes the gyrophase average, i.e., $\left\langle\delta \psi_{g_{c}}\right\rangle \equiv(2 \pi)^{-1} \oint d \theta \delta \psi(\mathbf{R}+\boldsymbol{\rho}, t)$, for instance, $d S_{1}=e \Omega^{-1}\left[\delta \psi_{g c}-\left\langle\delta \psi_{g c}\right\rangle\right] d \bar{\theta}$ has been chosen according to the gyrokinetic ordering. ${ }^{4}$

The second-order perturbation analysis deals with the equation

$$
\Gamma_{2}=\gamma_{2}-L_{1} \gamma_{1}+\left(\frac{1}{2} L_{1}^{2}-L_{2}\right) \gamma_{0}+d S_{2}
$$

The resulting equations have not been utilized in most practical applications. ${ }^{67-72}$ However, the resulting quadratic lowfrequency ponderomotive like terms are required for energy conservation up to $O\left(\epsilon_{\phi}^{2}\right)$, in the formulation in terms of the total distribution function. ${ }^{3,4}$ The importance of keeping those terms for theoretical completeness has been discussed in detail in a recent review article. ${ }^{7}$ The main result of the second order perturbation analysis is the nonlinear modification of the effective potential, which is described below. The derivation is quite similar to that of Ref. 4 and is not repeated here.

Finally, the total phase-space Lagrangian is given by

$$
\Gamma=\left(\frac{e}{c} \mathbf{A}+M \mathbf{u}_{E}+\bar{p}_{\|} \mathbf{b}\right) \cdot d \overline{\mathbf{R}}+\frac{\bar{\mu} B}{\Omega} d \bar{\theta}-\left(\bar{H}_{0}+e \delta \psi_{g y}\right) d t,
$$

where the effective gyrocenter perturbation potential is

$$
e \delta \psi_{g y} \equiv e\left\langle\delta \psi_{g c}\right\rangle+\frac{e^{2}}{2 M c^{2}}\left\langle\left|\delta \mathbf{A}_{g c}\right|^{2}\right\rangle-\frac{e^{3}}{2 M c \Omega} \frac{\partial}{\partial \mu}\left\langle\delta \tilde{\psi}_{g c}^{2}\right\rangle
$$

with $\delta \widetilde{\psi}_{g c} \equiv \delta \psi_{g c}-\left\langle\delta \psi_{g c}\right\rangle, \delta \widetilde{\Psi}_{g c} \equiv \int d \bar{\theta} \delta \tilde{\psi}_{g c}$, and the overbar is used for the gyrocenter variables. Here, the last term reduces to $-\left(T_{i} / 2\right) \delta u_{E}^{2} / v_{T i}^{2}$ in the long wavelength electrostatic limit. $^{65,73}$ We also find that the expression $\sim c / e \Omega\left\langle\nabla \delta \tilde{\Psi}_{g c} \times \mathbf{b} \cdot \nabla \delta \tilde{\psi}_{g c}\right\rangle$, which appeared in previous work $^{3-6}$ can be shown to be always smaller than the last term of Eq. (16) by at least an order $\delta_{B}$. The corresponding EulerLagrange equation can be obtained by using Eq. (3),

$$
-\frac{e}{c} \mathbf{B}^{*} \times \frac{d \overline{\mathbf{R}}}{d t}-\mathbf{b} \frac{d \bar{p}_{\|}}{d t}=\bar{\nabla}\left(\bar{H}_{0}+e \delta \psi_{g y}\right) .
$$

Equation (17) can be decomposed into the following gyrocenter equations of motion:

$$
\begin{aligned}
\frac{d \overline{\mathbf{R}}}{d t}= & \bar{v}_{\|} \frac{\mathbf{B}^{*}}{B_{\|}^{*}}+\frac{c \mathbf{b}}{e B_{\|}^{*}} \times\left[e \bar{\nabla}\left(\Phi+\delta \psi_{g y}\right)+\bar{\mu} \bar{\nabla} B\right. \\
& \left.+\frac{\bar{\mu} B}{2 \Omega} \bar{\nabla}\left(\mathbf{b} \cdot \bar{\nabla} \times \mathbf{u}_{E}\right)+\frac{M}{2} \bar{\nabla}\left(u_{E}^{2}\right)\right],
\end{aligned}
$$

and

$$
\begin{aligned}
\frac{d \bar{p}_{\|}}{d t}= & -\frac{\mathbf{B}^{*}}{B_{\|}^{*}} \cdot\left[e \bar{\nabla}\left(\Phi+\delta \psi_{g y}\right)+\bar{\mu} \bar{\nabla} B+\frac{\bar{\mu} B}{2 \Omega} \bar{\nabla}\left(\mathbf{b} \cdot \bar{\nabla} \times \mathbf{u}_{E}\right)\right. \\
& \left.+\frac{M}{2} \bar{\nabla}\left(u_{E}^{2}\right)\right]
\end{aligned}
$$

where the effective parallel velocity is

$$
\bar{v}_{\|}=\frac{\bar{p}_{\|}}{M}+e \frac{\partial \delta \psi_{g y}}{\partial \bar{p}_{\|}} .
$$

The $c \mathbf{b} / B_{\|}^{*} \times \bar{\nabla} \delta \psi_{g y}$ term on the R.H.S. of Eq. (18) contains terms which are responsible for turbulence-driven radial transport. These include:

i) electrostatic $\mathbf{E} \times \mathbf{B}$ transport contained in $c \mathbf{b} / B$ $\times \bar{\nabla}\left\langle\delta \phi_{g c}\right\rangle \simeq \delta \mathbf{u}_{E} ;$

ii) magnetic flutter transport which is proportional to $-\left(\bar{p}_{\|} / M\right) \mathbf{b} / B_{0} \times \bar{\nabla}\left\langle\delta A_{\| g c}\right\rangle \simeq\left(\bar{p}_{\|} / M\right) \delta \mathbf{B}_{\perp} / B$; and finally

iii) a compressional magnetic fluctuation driven piece $-\mathbf{b} / B \times \bar{\nabla}\left\langle\mathbf{c}_{\perp} \cdot \delta \mathbf{A}_{\perp}\right\rangle \simeq(c \bar{\mu} / e B) \mathbf{b} \times \bar{\nabla} \delta B_{\|}$, for $k_{\perp} \rho_{i} \ll 1$.

It is important to note that the electromagnetic character of the turbulence does not necessarily imply that the magnetic transport mechanisms described by items ii) and iii) are sig- 
nificant for self-consistent transport carried by collective fluctuations. ${ }^{74-76}$ Many details and subtleties involved in the electrostatic $\mathbf{E} \times \mathbf{B}$ drift were exhaustively discussed in Ref. 77.

\section{NONLINEAR GYROKINETIC VLASOV-MAXWELL SYSTEM}

With Eqs. (18) and (19), one can write the gyrokinetic Vlasov equation for the gyrocenter distribution function $F\left(\overline{\mathbf{R}}, \bar{\mu}, \bar{p}_{\|}, t\right)$,

$$
\frac{\partial F}{\partial t}+\frac{d \overline{\mathbf{R}}}{d t} \cdot \bar{\nabla} F+\frac{d \bar{p}_{\|}}{d t} \frac{\partial F}{\partial \bar{p}_{\|}}=0 .
$$

Here, $d \bar{\mu} / d t \equiv 0$ and $\partial F / \partial \bar{\theta} \equiv 0$ have been used. One can also write the gyrokinetic Vlasov equation in the following continuity equation form:

$$
\frac{\partial\left(B_{\|}^{*} F\right)}{\partial t}+\bar{\nabla} \cdot\left(B_{\|}^{*} \frac{d \overline{\mathbf{R}}}{d t} F\right)+\frac{\partial}{\partial \bar{p}_{\|}}\left(B_{\|}^{*} \frac{d \bar{p}_{\|}}{d t} F\right)=0 .
$$

This is possible because Eqs. (18) and (19) satisfy the following phase-space conservation law:

$$
\bar{\nabla} \cdot\left(B_{\|}^{*} \frac{d \overline{\mathbf{R}}}{d t}\right)+\frac{\partial}{\partial \bar{p}_{\|}}\left(B_{\|}^{*} \frac{d \bar{p}_{\|}}{d t}\right)=0 .
$$

The continuity equation form is sometimes more useful in applications which involve taking velocity moments. ${ }^{78-80}$

We note that various extensions of the gyrokinetic Vlasov equation are conceptually straightforward, once one decides on the generalized ordering. This is because a systematic phase-space Lagrangian derivation of guiding center drift is available, for instance, from Refs. 36-38. On the other hand, expressing the particle charge density and current in terms of the gyrocenter distribution function in the gyrokinetic Maxwell's equations involves a rather cumbersome pullback transformation from the gyrocenter coordinate to the particle coordinate. Indeed, in many cases, the gyrokinetic Maxwell's equations were only presented in their most general form representing the pullback transformation, without an explicit evaluation of the integrals. However, for many relevant applications including gyrokinetic simulations, explicit evaluations of the particle charge density and current from the gyrocenter distribution function are necessary. This important aspect of explicit representation in the gyrokinetic Poisson equation was recognized and carried out $^{2}$ before the more rigorous Hamiltonian method and the phase-space Lagrangian method were introduced in the nonlinear gyrokinetic formalism. ${ }^{3,4}$ Consideration of energy conservation between particles and fields, in particular, identifying the energy invariant in the gyrokinetic Vlasov-Maxwell system also requires the same level of explicitness in the gyrokinetic Maxwell's equations and the gyrokinetic Vlasov equation. Regarding this, the systematic derivation was first achieved in Ref. 3 for electrostatic fluctuations in a straight magnetic field, and then extended to toroidal geometry ${ }^{4}$ and to electromagnetic fluctuations, ${ }^{5,6}$ and finally to the presence of strongly sheared $\mathbf{E} \times \mathbf{B}$ flows. ${ }^{24}$ It is important to treat the gyrokinetic Vlasov equation, the gyrokinetic Maxwell's equations, and the energy invariant self-consistently on an equal footing. These form three pillars ${ }^{7}$ of nonlinear gyrokinetic theory. We note that a field theoretical variational derivation was also introduced. ${ }^{73,81}$

Now, we present the gyrokinetic Maxwell equations in which the ion particle charge density and current are expressed in terms of the gyrocenter distribution function $F\left(\overline{\mathbf{R}}, \bar{\mu}, \bar{p}_{\|}, t\right)$,

$$
\begin{aligned}
\nabla^{2}(\Phi+\delta \phi)= & -4 \pi e\left[\int d ^ { 6 } \overline { \mathbf { Z } } \left(F+g_{1}^{\bar{\mu}} \frac{\partial F}{\partial \bar{\mu}}+g_{1}^{\overline{\mathbf{R}}} \cdot \bar{\nabla} F\right.\right. \\
& \left.\left.+g_{1}^{\overline{p_{1}}} \frac{\partial F}{\partial \bar{p}_{\|}}\right) \delta^{3}(\overline{\mathbf{R}}-\mathbf{x}+\overline{\boldsymbol{\rho}})-n_{e}(\mathbf{x}, t)\right],
\end{aligned}
$$

$$
\begin{aligned}
\nabla^{2}(\mathbf{A}+\delta \mathbf{A})= & -\frac{4 \pi e}{c}\left[\int d^{6} \overline{\mathbf{Z}}\left(\frac{\bar{p}_{\|}}{M} \mathbf{b}+\mathbf{u}_{E}+\mathbf{c}_{\perp}\right)\right. \\
& \times\left(F+\bar{g}_{1}^{\mu} \frac{\partial F}{\partial \bar{\mu}}+\bar{g}_{1}^{\mathbf{R}} \cdot \bar{\nabla} F+g_{1} \bar{p}_{\|} \frac{\partial F}{\partial \bar{p}_{\|}}\right) \\
& \left.\times \delta^{3}(\overline{\mathbf{R}}-\mathbf{x}+\overline{\boldsymbol{\rho}})-\int d^{3} \mathbf{v} \mathbf{v} f_{e}(\mathbf{x}, \mathbf{v}, t)\right],
\end{aligned}
$$

where the Coulomb gauge $\nabla \cdot(\mathbf{A}+\delta \mathbf{A})=0$ was used, and $d^{6} \overline{\mathbf{Z}} \equiv\left(B_{\|}^{*} / M^{2}\right) d^{3} \overline{\mathbf{R}} d \bar{p}_{\|} d \bar{\mu} d \bar{\theta}, g_{1}^{\bar{\mu}}=(e / M c)$ $\times\left(e / c \delta \mathbf{A}_{g c} \cdot \partial \bar{\rho} / \partial \bar{\theta}+\partial S_{1} / \partial \bar{\theta}\right), \quad g_{1}^{\overline{\mathbf{R}}}=-\left(1 / B_{\|}^{*}\right) \mathbf{b} \times\left(\delta \mathbf{A}_{g c}\right.$ $\left.+c / e \bar{\nabla} S_{1}\right), g_{1}^{\bar{p}_{\|}}=\mathbf{B}^{*} / B_{\|}^{*} \cdot\left(e / c \delta \mathbf{A}_{g c}+\bar{\nabla} S_{1}\right), \bar{p}_{\|} \simeq p_{\|}+(e / c) \delta A_{\| g c}$, and $S_{1}=e \Omega^{-1} \int d \bar{\theta}\left(\delta \psi_{g c}-\left\langle\delta \psi_{g c}\right\rangle\right)$. Since the LHS is $\nabla \times \mathbf{B}$, the RHS should be divergence-free. Although we do not verify this explicitly here, it is an important property ${ }^{73}$ in relation to the Darwin model. ${ }^{82}$ It is encouraging to note that Alfvén modes have been successfully simulated ${ }^{83}$ using a simpler set of equations. ${ }^{4,5}$ It is also important to note that in our Hamiltonian formulation, $\bar{p}_{\|}$is a canonical momentum including $(e / c) \mathbf{b}^{*} \cdot \delta \mathbf{A}$. In this case, our formulation is closer to the $p_{z}$ formulation in Ref. 5 than to the $v_{z}$ formulation in the same work. In Eq. (23), the first four terms on the right-hand side are the ion particle density $n_{i}(\mathbf{x}, t)$, written in terms of the gyrocenter distribution function. The first term is the gyroaveraged gyrocenter density contribution, while the other three terms are the general expression for the polarization density. It can be shown that the $g_{1}^{\overline{\mathbf{R}}}$ term and $g_{1}^{\bar{p}_{\|}}$term are smaller than $g_{1}^{\bar{\mu}}$ term by an order, so these two terms can be neglected in Eqs. (23) and (24) as well. The global gyrokinetic Vlasov-Maxwell energy is obtained by the Noether method and integration over space, as described in Eq. (50) of Ref. 81, and Eq. (199) of Ref. 7, 


$$
\begin{aligned}
E= & \frac{1}{8 \pi} \int d^{3} \mathbf{x}\left[|\nabla(\Phi+\delta \phi)|^{2}+\left|\mathbf{B}_{0}+\delta \mathbf{B}\right|^{2}\right] \\
& +\int d^{6} \mathbf{z} f_{e}(\mathbf{z}) \frac{1}{2} m_{e} \mathbf{v}^{2}+\int d^{6} \overline{\mathbf{Z}} F_{i}\left\{\bar{\mu} B+\bar{p}_{\|}^{2} /(2 M)\right. \\
& +\frac{M}{2} u_{E}^{2}+\frac{\bar{\mu} B}{2 \Omega} \mathbf{b} \cdot \bar{\nabla} \times \mathbf{u}_{E}-\frac{1}{c}\left(\left\langle\delta \mathbf{A}_{g c}\right\rangle \cdot \mathbf{u}^{*}\right. \\
& \left.+\left\langle\delta \mathbf{A}_{g c} \cdot \mathbf{c}_{\perp}\right\rangle\right)+\frac{e^{2}}{2 M c^{2}}\left\langle\left|\delta \mathbf{A}_{g c}\right|^{2}\right\rangle+\frac{e^{3}}{2 M c \Omega} \frac{\partial}{\partial \mu} \\
& \left.\times\left[\left\langle\delta \widetilde{\phi}_{g c}^{2}\right\rangle-\frac{1}{c^{2}}\left\langle\left(\delta \widetilde{\mathbf{A}}_{g c} \cdot \mathbf{u}^{*}+\delta \mathbf{A}_{g c} \cdot \mathbf{c}_{\perp}\right)^{2}\right\rangle\right]\right\},
\end{aligned}
$$

where $\mathbf{u}^{*}=\bar{p}_{\|} / M \mathbf{b}+\mathbf{u}_{E}, \quad \widetilde{\boldsymbol{\alpha}}=\int d \theta \delta \tilde{\mathbf{A}} g c$, and $\tilde{\beta}=\int d \theta \delta \widetilde{\mathbf{A}_{g c}} \cdot \mathbf{c}_{\perp}$. We note that the terms $\sim\left\langle\bar{\nabla} \delta \widetilde{\Phi}_{g c} \cdot \mathbf{b} \times \bar{\nabla} \delta \widetilde{\phi}_{g c}-1 / c^{2} \bar{\nabla}\left(\widetilde{\boldsymbol{\alpha}} \cdot \mathbf{u}^{*}\right.\right.$ $\left.+\widetilde{\beta}) \cdot \mathbf{b} \times \bar{\nabla}\left(\delta \widetilde{\mathbf{A}}_{g c} \cdot \mathbf{u}^{*}+\delta \widetilde{\mathbf{A}_{g c}} \cdot \mathbf{c}_{\perp}\right)\right\rangle$, which are corresponding to the $g_{1}^{\overline{\mathbf{R}}}$ term in Poisson's equation are also smaller than the other terms by at least one order, so they are also neglected to conserve the energy exactly.

Here, we do not specify the electron dynamics, so as to write the electron kinetic energy in a primitive form. However, depending on the problem considered, either a drift kinetic equation $^{84}$ or a bounce-averaged kinetic equation ${ }^{85,86}$ can be used when it is appropriate. Sometimes, nonlinearity associated with the magnetically trapped electrons is important. ${ }^{87-90}$ In Eq. (25), the last term represents the sloshing energy. ${ }^{3}$ In the total $F$ formulation, the second-order nonlinear correction to the effective potential [the last term in Eq. (16) which leads to the ponderomotive force] should be kept alongside the sloshing energy in Eq. (25) in order to ensure energy conservation. Here, $\mathbf{E} \equiv-\nabla(\Phi+\delta \phi)$ is the total electric field, and $\mathbf{B}=\mathbf{B}_{0}+\delta \mathbf{B}$ is the total magnetic field. Equation (25) is a generalization of the result of Ref. 24 to the fully electromagnetic case. Now, we consider limiting cases.

\section{A. Long wavelength expression for arbitrary $\boldsymbol{F}$}

In the long wavelength limit $k_{\perp} \rho_{i} \ll 1$, the gyrokinetic Vlasov equation is

$$
\frac{\partial F}{\partial t}+\frac{d \overline{\mathbf{R}}}{d t} \cdot \bar{\nabla} F+\frac{d \bar{p}_{\|}}{d t} \frac{\partial F}{\partial \bar{p}_{\|}}=0,
$$

with corresponding equations of motion,

$$
\begin{aligned}
\frac{d \overline{\mathbf{R}}}{d t}= & \bar{v}_{\|} \frac{\mathbf{B}^{*}}{B_{\|}^{*}}+\frac{c \mathbf{b}}{e B_{\|}^{*}} \times\left[e \bar{\nabla}\left(\Phi+\delta \psi_{g y}\right)+\bar{\mu} \bar{\nabla} B\right. \\
& \left.+\frac{\bar{\mu} B}{2 \Omega} \bar{\nabla}\left(\mathbf{b} \cdot \bar{\nabla} \times \mathbf{u}_{E}\right)+\frac{M}{2} \bar{\nabla}\left(u_{E}^{2}\right)\right],
\end{aligned}
$$

and

$$
\begin{aligned}
\frac{d \bar{p}_{\|}}{d t}= & -\frac{\mathbf{B}^{*}}{B_{\|}^{*}} \cdot\left[e \bar{\nabla}\left(\Phi+\delta \psi_{g y}\right)+\bar{\mu} \bar{\nabla} B\right. \\
& \left.+\frac{\bar{\mu} B}{2 \Omega} \bar{\nabla}\left(\mathbf{b} \cdot \bar{\nabla} \times \mathbf{u}_{E}\right)+\frac{M}{2} \bar{\nabla}\left(u_{E}^{2}\right)\right] .
\end{aligned}
$$

But the effective gyrocenter perturbation potential in Eq. (27) and (28) can be given in simplified form as

$$
\begin{aligned}
e \delta \psi_{g y} \simeq & e\left\langle\delta \psi_{g c}\right\rangle+\frac{e^{2}}{2 M c^{2}} \delta A_{\|}^{2}-\frac{M}{2}\left|\delta \mathbf{u}_{E}^{\prime}+\frac{\bar{p}_{\|}}{M} \frac{\delta \mathbf{B}_{\perp}}{B}\right|^{2} \\
& -\frac{e}{c}\left(\delta \mathbf{u}_{E}^{\prime}+\frac{\bar{p}_{\|}}{M} \frac{\delta \mathbf{B}_{\perp}}{B}\right) \cdot \delta \mathbf{A}_{\perp},
\end{aligned}
$$

where $\quad \delta \mathbf{u}_{E}^{\prime}=c / B \mathbf{b} \times \nabla_{\perp} \delta \phi^{\prime} \quad$ and $\quad \nabla_{\perp} \delta \phi^{\prime}=\nabla_{\perp} \delta \phi$ $-1 / c\left(\nabla_{\perp} \delta \mathbf{A}\right) \cdot \mathbf{u}_{E}$. Also in this limit, the effective parallel velocity from Eq. (20) is

$$
\begin{aligned}
\bar{v}_{\|}= & \frac{\bar{p}_{\|}}{M}\left(1-\frac{\delta B_{\perp}^{2}}{B^{2}}\right)-\frac{e}{M c}\left\langle\delta A_{\| g c}\right\rangle \\
& -\frac{\delta \mathbf{B}_{\perp}}{B} \cdot\left(\delta \mathbf{u}_{E}^{\prime}+\frac{e}{M c} \delta \mathbf{A}_{\perp}\right) .
\end{aligned}
$$

The gyrokinetic Maxwell equations (23) and (24) become

$$
\begin{aligned}
& \nabla^{2}(\Phi+\delta \phi)=-4 \pi e\left\{\bar{N}_{i}(\mathbf{x}, t)-n_{e}(\mathbf{x}, t)+\frac{\delta B_{\|}}{B} N_{i}\right. \\
& +\frac{e}{M} \nabla_{\perp} \cdot\left(\frac{N_{i}}{\Omega_{i}^{2}} \nabla_{\perp} \delta \phi^{\prime}\right) \\
& \left.-\frac{1}{M c} \nabla_{\perp} \cdot\left(\frac{J_{i \|}}{\Omega_{i}^{2}} \nabla_{\perp} \delta A_{\|}\right)\right\} \text {, } \\
& \nabla^{2}\left(A_{\|}+\delta A_{\|}\right)=-\frac{4 \pi}{c}\left\{\bar{J}_{i \|}(\mathbf{x}, t)-j_{e \|}(\mathbf{x}, t)+\frac{\delta B_{\|}}{B} J_{i \|}\right. \\
& +\frac{e}{M} \nabla_{\perp} \cdot\left(\frac{J_{i \|}}{\Omega_{i}^{2}} \nabla_{\perp} \delta \phi^{\prime}\right) \\
& \left.-\frac{e^{2}}{M c} \nabla_{\perp} \cdot\left(\frac{\Pi_{i \|}}{\Omega_{i}^{2}} \nabla_{\perp} \delta A_{\|}\right)\right\} \text {, } \\
& \nabla^{2}\left(\mathbf{A}_{\perp}+\delta \mathbf{A}_{\perp}\right)=-\frac{4 \pi}{c}\left\{\overline{\mathbf{J}}_{i \perp}(\mathbf{x}, t)+\overline{\mathbf{J}}_{i E}(\mathbf{x}, t)-\mathbf{j}_{e \perp}(\mathbf{x}, t)\right. \\
& -\mathbf{j}_{e E}(\mathbf{x}, t)+\frac{\delta B_{\|}}{B} \mathbf{J}_{i E}+\frac{e^{2}}{M} \nabla_{\perp} \\
& \cdot\left[\frac{N_{i}}{\Omega_{i}^{2}}\left(\nabla_{\perp} \delta \phi^{\prime}\right) \mathbf{u}_{E}\right]-\frac{e}{M c} \nabla_{\perp} \\
& \cdot\left[\frac{J_{i \|}}{\Omega_{i}^{2}}\left(\nabla_{\perp} \delta A_{\|}\right) \mathbf{u}_{E}\right] \\
& +e N_{i}\left[\delta \mathbf{u}_{E}-\frac{\delta B_{\|}}{B}\left(\mathbf{u}_{E} \cdot \delta \hat{\mathbf{A}}_{\perp}\right) \delta \hat{\mathbf{A}}_{\perp}\right] \\
& \left.+\frac{J_{i \|}}{B} \delta \mathbf{B}_{\perp}-2 c \nabla \times\left(\frac{P_{\perp} \delta B_{\|}}{B^{2}} \mathbf{b}\right)\right\},
\end{aligned}
$$


where $\delta \mathbf{u}_{E}=(c / B) \mathbf{b} \times \nabla_{\perp} \delta \phi$ and $\delta \hat{\mathbf{A}}_{\perp}=\delta \mathbf{A}_{\perp} /\left|\delta \mathbf{A}_{\perp}\right|$. Noting an apparent symmetry, Eqs. (31) and (32) can be written using a two vector notation,

$$
\begin{aligned}
\nabla^{2}\left(A^{\alpha}+\delta A^{\alpha}\right)= & -\frac{4 \pi}{c}\left\{\bar{J}_{i}^{\alpha}(\mathbf{x}, t)-j_{e}^{\alpha}(\mathbf{x}, t)+\frac{\delta B_{\|}}{B} J_{i}^{\alpha}\right. \\
& \left.+\frac{e}{M} \nabla_{\perp} \cdot\left[\frac{\Pi_{i}^{\prime \alpha \beta}}{\Omega_{i}^{2}} \nabla_{\perp} \delta A_{\beta}^{\prime}\right]\right\},
\end{aligned}
$$

where $A^{\alpha}=\left(\Phi, A_{\|}\right), J^{\alpha}=\left(c e N, J_{\|}\right), \nabla_{\perp} \delta A^{\prime \alpha}=\left(\nabla_{\perp} \delta \phi^{\prime}, \nabla_{\perp} \delta A_{\|}\right)$, and $\Pi_{i}^{\prime \alpha \beta}=\left(\begin{array}{c}c e N_{i} J_{i \|} \\ J_{i \|} e \Pi_{i} / c\end{array}\right)$, the metric tensor $g_{\alpha \beta}=\left(\begin{array}{cc}1 & 0 \\ 0-1\end{array}\right)$.

Equations (29) and (31) reduce to those of Ref. 24 in the electrostatic limit. They also reduce to those of Ref. 7 in the absence of $\mathbf{u}_{E}$. Here, we note that a coefficient $n / \Omega^{2} \propto n / B^{2}$ appears inside the divergence operator $\left(\nabla_{\perp}\right)$ in the last two terms of Eqs. (31) and (32), and the sixth and seventh terms on the R.H.S. of Eq. (33). These expressions are more general than those from Refs. 7 and 24 in toroidal geometry where the $1 / B^{2}$ factor appears outside the divergence operator $\left(\nabla_{\perp}\right)$. Since we assume that $L_{B} \gg L_{p}, L_{E}$ here (and in other references) the resulting modification is minor, quantitatively. However this general expression is physically appealing, since $v_{A}^{2} \propto B^{2} / n$ and a close relation exists between the polarization density and the vorticity which appears in reduced fluid equations. ${ }^{65}$ This could serve as a useful guideline when one wants to extend the formulation to a more compact confinement device, such as, NSTX. ${ }^{52}$ The third term on the R.H.S. of Eq. $(31),\left(\delta B_{\| k} / B\right) N_{i}$, can be shown to originate from the $\mathbf{E} \times \mathbf{B}$ drift caused by an induction electric field perpendicular to $\mathbf{B}_{0}$, i.e., $\delta \mathbf{E}_{\perp \text { ind }}=-(1 / c) \partial / \partial t \delta \mathbf{A}_{\perp}$.

Now, we discuss the shielding properties of gyrokinetic plasma in the presence of the equilibrium electric field. As widely recognized, representing the polarization drift as a shielding term in the gyrokinetic Poisson equation ${ }^{2}$ has provided one of the key computational advantages of the gyrokinetic approach. However, there is an important qualitative difference between the Debye shielding and the polarization shielding, in addition to their magnitudes. In Eq. (31), the Debye shielding term on the left-hand side contains both the equilibrium potential and the perturbed potential, while the polarization density involves $\delta \phi$ only. This is because the polarization density is related to the polarization drift via the continuity equation, and the polarization drift occurs only for the time-varying electric field. Here, it is shown that if one uses a simplified definition of a gyrocenter density, a polarization-density-like term associated with $\Phi$ appears. ${ }^{24}$ The gyroaveraged gyrocenter density $\bar{N}_{i}$ is defined by

$$
\bar{N}_{i} \equiv \int \frac{B_{\|}^{*}}{M^{2}} d^{3} \overline{\mathbf{R}} d \bar{p}_{\|} d \bar{\theta} d \bar{\mu} F_{i} \delta^{3}(\overline{\mathbf{R}}-\mathbf{x}+\overline{\boldsymbol{\rho}}),
$$

and this differs slightly from the gyrocenter density $N_{i}$, which is defined by

$$
N_{i} \equiv \int \frac{B_{\|}^{*}}{M^{2}} d^{3} \overline{\mathbf{R}} d \bar{p}_{\|} d \bar{\theta} d \bar{\mu} F_{i} \delta^{3}(\overline{\mathbf{R}}-\mathbf{x}) .
$$

In computation, it has been the usual practice to use a simplified gyrocenter density $\bar{N}_{i}^{0}$ with an approximate Jacobian of a phase-space volume element $B$ instead,

$$
\bar{N}_{i}^{0} \equiv \int \frac{B}{M^{2}} d^{3} \overline{\mathbf{R}} d \bar{p}_{\|} d \bar{\theta} d \bar{\mu} F_{i} \delta^{3}(\overline{\mathbf{R}}-\mathbf{x}+\overline{\boldsymbol{\rho}}) .
$$

Then, using the approximation, $B_{\|}^{*} \equiv B[1+\mathbf{b} / \Omega$ $\left.\cdot \nabla \times\left(\mathbf{u}_{E}+\bar{p}_{\|} / M \mathbf{b}\right)\right] \simeq B+(1 / \Omega) \nabla_{\perp}^{2} \Phi$, one can write Eq. (35) as

$$
\bar{N}_{i} \simeq \bar{N}_{i}^{0}\left(1+\frac{e \rho_{s}^{2}}{T_{e}} \nabla_{\perp}^{2} \Phi\right) .
$$

Now, the last term looks like the polarization density associated with $\Phi$. This identification might be useful when one tries to perform a long-time simulation with a slowly time varying $\Phi .^{91-95}$

In passing, we remark that it is possible to formulate gyrokinetics in such a way that the polarization drift appears in the gyrokinetic Vlasov equation ${ }^{96}$ rather than as a polarization density in the gyrokinetic Poisson equation. In Refs. $33-35$, the polarization drift associated with a time-varying background appears in the gyrokinetic Vlasov equation, while the polarization density associated with shorter wavelength fluctuations appears in the gyrokinetic Poisson equation as usual. If one tries to describe a transport barrier formation, such as, an H-mode transition, a distinction between the time-varying background and large-scale fluctuations becomes rather subtle. In addition, a division between large scale fluctuations and short scale fluctuations seems arbitrary, as noted in previous work. ${ }^{66}$ Other terms which appear in Eqs. (31)-(33) are defined as

$$
\begin{aligned}
&\left(\bar{J}_{i \|}, \overline{\mathbf{J}}_{i E}, \overline{\mathbf{J}}_{i \perp}\right) \equiv e \int \frac{B_{\|}^{*}}{M^{2}} d^{3} \overline{\mathbf{R}} d \bar{p}_{\|} d \bar{\theta} d \bar{\mu} F_{i}\left(\frac{\bar{p}_{\|}}{M}, \mathbf{u}_{E}, \mathbf{c}_{\perp}\right) \\
& \times \delta^{3}(\overline{\mathbf{R}}-\mathbf{x}+\overline{\boldsymbol{\rho}}), \\
&\left(j_{e \|}, \mathbf{j}_{e E}, \mathbf{j}_{e \perp}\right) \equiv e \int d^{3} \mathbf{v} f_{e} \mathbf{x},\left(\bar{v}_{\|}, \mathbf{u}_{E}, \mathbf{c}_{\perp}\right), \\
&\left(J_{i \|}, \mathbf{J}_{i E}\right) \equiv e \int \frac{B_{\|}^{*}}{M^{2}} d^{3} \overline{\mathbf{R}} d \bar{p} \| d \bar{\theta} d \bar{\mu} F_{i}\left(\frac{\bar{p} \|}{M}, \mathbf{u}_{E}\right) \delta^{3}(\overline{\mathbf{R}}-\mathbf{x}), \\
& \Pi_{i \|} \equiv \int \frac{B_{\|}^{*}}{M^{2}} d^{3} \overline{\mathbf{R}} d \bar{p}_{\|} d \bar{\theta} d \bar{\mu} F_{i} \frac{\bar{p}_{\|}^{2}}{2 M} \delta^{3}(\overline{\mathbf{R}}-\mathbf{x}), \\
& P_{\perp} \equiv \int \frac{B_{\|}^{*}}{M^{2}} d^{3} \overline{\mathbf{R}} d \bar{p}_{\|} d \bar{\theta} d \bar{\mu} F_{i} \bar{\mu} B \delta^{3}(\overline{\mathbf{R}}-\mathbf{x}) .
\end{aligned}
$$

Since $\bar{v}_{\|} \simeq \bar{p}_{\|} / m_{e}+\left(e / m_{e} c\right) \delta A_{\|}$, the collisionless skin depth term $\left(\omega_{p e} / c\right)^{2} \delta A_{\|}$will appear explicitly on the R.H.S. of Eq. (32), if the current density $j_{e \|}$ is defined by the moment of $\bar{p}_{\|}$. We can see that the electrons contribute to the collisionless 
skin depth term dominantly, so it is reasonable to neglect that of the ions in Eq. (24). Turbulence at the scale of the collisionless skin depth was simulated ${ }^{97,98}$ and measured in experiments. $^{99}$
The corresponding energy invariant which is exactly conserved by Eqs. (26)-(33) which are long wavelength approximations to gyrokinetic Vlasov equation and Maxwell equations, respectively, is

$$
\begin{aligned}
E= & \frac{1}{8 \pi} \int d^{3} \mathbf{x}\left[|\nabla(\Phi+\delta \phi)|^{2}+\left|\mathbf{B}_{0}+\delta \mathbf{B}\right|^{2}\right]+\int d^{6} \mathbf{z} f_{e}(\mathbf{z}) \frac{1}{2} m_{e} \mathbf{v}^{2}+\int d^{6} \overline{\mathbf{Z}} F_{i}\left(\bar{\mu} B+\frac{M}{2} u_{E}^{2}+\frac{\bar{p}_{\|}^{2}}{2 M}+\frac{\bar{\mu} B}{2 \Omega} \mathbf{b} \cdot \bar{\nabla} \times u_{E}\right. \\
& -\frac{1}{c}\left(\left\langle\delta \mathbf{A}_{g c}\right\rangle \cdot \mathbf{u}^{*}+\left\langle\delta \mathbf{A}_{g c} \cdot \mathbf{c}_{\perp}\right\rangle\right)+\frac{e^{2}}{2 M c^{2}}\left(\delta A_{\|}\right)^{2}+\frac{M}{2}\left\{\delta \mathbf{u}_{E}^{2}-\left[\frac{\bar{p}_{\|}}{M} \frac{\delta \mathbf{B}_{\perp}}{B}-\frac{\delta B_{\|}}{B}\left(\mathbf{u}_{E} \cdot \delta \hat{\mathbf{A}}_{\perp}\right) \delta \hat{\mathbf{A}}\right]_{\perp}^{2}\right\} \\
& \left.-\frac{e}{c B}\left(\frac{\bar{p}_{\|}}{M} \delta \mathbf{B}_{\perp}-\frac{\delta B_{\|}}{B} \mathbf{u}_{E}\right) \cdot \delta \mathbf{A}\right) .
\end{aligned}
$$

\section{B. Arbitrary wavelength expressions for Maxwellian $F$ in $\bar{\mu}$}

Now, we consider arbitrary values of $k_{\perp} \rho_{i}$, but assume that $F$ is Maxwellian in $\bar{\mu}$ [i.e., $F \propto \exp (-\bar{\mu} B / T)$ ]. Note that this approximation is a bit more general than a linearization with a Maxwellian equilibrium distribution function $F_{0}$, but inclusive of the latter. In this limit, after expanding in Fourier components, Eqs. (23) and (24) become

$$
\begin{aligned}
\nabla^{2}(\Phi+\delta \phi)=- & 4 \pi e\left\{\bar{N}_{i}-n_{e}-\sum_{k} \exp (i \mathbf{k} \cdot \mathbf{x})\left[\frac{1-\Gamma_{0}}{T_{i}}\left(e N_{i} \delta \phi_{k}^{\prime}-\frac{1}{c} J_{i \|} \delta A_{\| k}\right)+\left(\Gamma_{1}-\Gamma_{0}\right) \frac{\delta B_{\| k}}{B} N_{i}\right]\right\}, \\
\nabla^{2}\left(A_{\|}+\delta A_{\|}\right)=- & \frac{4 \pi}{c}\left\{\bar{J}_{i \|}-j_{e \|}-\sum_{k} \exp (i \mathbf{k} \cdot \mathbf{x})\left[\frac{e}{T_{i}}\left(1-\Gamma_{0}\right)\left(J_{i \|} \delta \phi_{k}^{\prime}-\frac{e}{c} \Pi_{i \|} \delta A_{\| k}\right)+\left(\Gamma_{1}-\Gamma_{0}\right) \frac{\delta B_{\| k}}{B} J_{i \|}\right]\right\}, \\
\nabla^{2}\left(\mathbf{A}_{\perp}+\delta \mathbf{A}_{\perp}\right)= & -\frac{4 \pi}{c}\left(\overline{\mathbf{J}}_{i \perp}+\overline{\mathbf{J}}_{i E}-\mathbf{j}_{e \perp}-\mathbf{j}_{e E}-\sum_{k} \exp (i \mathbf{k} \cdot \mathbf{x})\left[\frac{e}{T_{i}}\left(1-\Gamma_{0}\right)\left(e N_{i} \delta \phi_{k}^{\prime}-\frac{1}{c} J_{i \|} \delta A_{\| k}\right) \mathbf{u}_{E}+\left(\Gamma_{1}-\Gamma_{0}\right) \frac{\delta B_{\| k}}{B} \mathbf{j}_{E}\right.\right. \\
& \left.\left.+\left(\Gamma_{1}-\Gamma_{0}\right)\left\{e N_{i}\left[\delta \mathbf{u}_{E k}-\left(\mathbf{u}_{E} \cdot \delta \hat{\mathbf{A}}_{\perp k}\right) \delta \hat{\mathbf{A}}_{\perp k}\right]+J_{i \|} \frac{\delta \mathbf{B}_{\perp k}}{B}\right\}+\frac{e^{2}}{M c} N_{i}\left[2 \Gamma_{1}+k_{\perp}^{2} \rho_{i}^{2}\left(\Gamma_{0}+\Gamma_{2}-2 \Gamma_{1}\right)\right] \delta \mathbf{A}_{\perp k}\right]\right),
\end{aligned}
$$

where $\delta \phi_{k}^{\prime}=\delta \phi_{k}-(1 / c) \mathbf{u}_{E} \cdot \delta \mathbf{A}_{\perp k}$. We do not keep some higher order terms kept in Refs. 3 and 5, since those are not necessary for energy conservation. Once again, Eqs. (40) and (41) can be combined in a covariant fashion with a two-vector notation,

$$
\begin{aligned}
\nabla^{2}\left(A^{\alpha}+\delta A^{\alpha}\right)= & -\frac{4 \pi}{c}\left\{\bar{J}_{i}^{\alpha}-j_{e}^{\alpha}-\sum_{k} \exp (i \mathbf{k} \cdot \mathbf{x})\left[\frac{e}{T_{i}}\left(1-\Gamma_{0}\right) \Pi_{i}^{\prime \alpha \beta} \delta A_{\beta k}^{\prime}+\left(\Gamma_{1}-\Gamma_{0}\right) \frac{\delta B_{\| k}}{B} J_{i}^{\alpha}\right.\right. \\
& \left.\left.+\frac{e}{T_{i}}\left(\Gamma_{1}-\Gamma_{0}\right) i \rho_{i}^{2} \mathbf{k}_{\perp} \cdot\left(\nabla_{\perp} \Pi_{i}^{\prime \alpha \beta}\right) \delta A_{\beta k}^{\prime}\right]\right\},
\end{aligned}
$$

where $\Gamma_{n}(b)=I_{n}(b) e^{-b}$, and $I_{n}$ is the modified Bessel function of order $n$, where $b=k_{\perp}^{2} \rho_{i}^{2}$. The corresponding exactly conserved energy for this system can be written as

$$
\begin{aligned}
E= & \frac{1}{8 \pi} \int d^{3} \mathbf{x}\left[|\nabla(\Phi+\delta \phi)|^{2}+|\mathbf{B}+\delta \mathbf{B}|^{2}\right]+\int d^{6} \mathbf{z} f_{e}(\mathbf{z}) \frac{1}{2} m_{e}\left(v_{\|} \mathbf{b}+\mathbf{c}_{\perp}+\mathbf{u}_{E}\right)^{2}+\int d^{6} \overline{\mathbf{Z}} F_{i}\left[\bar{\mu} B+\frac{\bar{p}_{\|}^{2}}{2 M}+\frac{M}{2} u_{E}^{2}+\frac{\bar{\mu} B}{2 \Omega} \mathbf{b} \cdot \bar{\nabla} \times u_{E}\right. \\
& \left.-\frac{1}{c}\left(\left\langle\delta \mathbf{A}_{g c}\right\rangle \cdot \mathbf{u}^{*}+\left\langle\delta \mathbf{A}_{g c} \cdot \mathbf{c}_{\perp}\right\rangle\right)\right]+\frac{e}{2 T_{i}} \sum_{k}\left(1-\Gamma_{0}\right)\left\{e n_{0}\left|\delta \phi_{k}\right|^{2}-\frac{1}{c^{2}}\left[e \pi_{i 0 \|}\left|\delta A_{\| k}\right|^{2}+\mathbf{u}_{E 0} \cdot \delta \mathbf{A}_{-k}\left(\mathbf{j}_{i 0 E} \cdot \delta \mathbf{A}_{k}+2 j_{i 0 \|} \delta A_{\| k}\right)\right]\right\} \\
& +\frac{e^{2} n_{0}}{2 M c^{2}} \sum_{k}\left\{\left|\delta A_{\| k}\right|^{2}+\left[2 \Gamma_{1}+k_{\perp}^{2} \rho_{i}^{2}\left(\Gamma_{0}+\Gamma_{2}-2 \Gamma_{1}\right)\right] \frac{\left|\delta B_{\| k}\right|^{2}}{k_{\perp}^{2}}\right\}-\frac{1}{c B} \sum_{k}\left(\Gamma_{0}-\Gamma_{1}\right) \delta B_{\|-k}\left(\mathbf{j}_{i 0 E} \cdot \delta \mathbf{A}_{k}+j_{i 0 \|} \delta A_{\| k}\right)
\end{aligned}
$$


with

$$
\begin{aligned}
& n_{0} \equiv \int \frac{B_{\|}^{*}}{M^{2}} d \bar{p}_{\|} d \bar{\theta} d \bar{\mu} F_{i 0}, \\
& \left(j_{i 0 \|}, \mathbf{j}_{i 0 E}\right) \equiv e \int \frac{B_{\|}^{*}}{M^{2}} d \bar{p}_{\|} d \bar{\theta} d \bar{\mu} F_{i 0}\left(\frac{\bar{p}_{\|}}{M}, \mathbf{u}_{E 0}\right), \\
& \pi_{i 0 \|} \equiv \int \frac{B_{\|}^{*}}{M^{2}} d \bar{p}_{\|} d \bar{\theta} d \bar{\mu} F_{i 0} \frac{\bar{p}_{\|}^{2}}{2 M} .
\end{aligned}
$$

Once again, Eqs. (43) and (44) are the fully electromagnetic generalization of the electrostatic results in Ref. 24 .

\section{ACKNOWLEDGMENTS}

We would like to acknowledge useful discussions with B. D. Scott, A. Brizard, C. S. Chang, L. Chen, P. H. Diamond, Y. Chen, G. Dif-Pradalier, W. Horton, S. Ku, W. W. Lee, Z. Lin, V. Naulin, S. E. Parker, J. Rasmussen, G. Rewoldt, Y. Sarazin, H. Sugama, W. M. Tang, L. Villard, W. X. Wang, H. Weitzner, X. Q. Xu, and M. Yagi.

This work was supported by the U.S. DOE SciDAC-FSP Center for Plasma Edge Simulation, and the U.S. DOE SciDAC Center for Gyrokinetic Particle Simulation of Turbulent Transport in Burning Plasmas (T.S.H.), the China Scholarship Council (L.W.), U.S. Department of Energy Contract No. DE-AC02-76-CHO-3073 (T.S.H., L.W.), and a financial subvention from the Danish Agency for Science, Technology and Innovation (FNU 272-06-0367) (J.M.).

${ }^{1}$ E. A. Frieman and L. Chen, Phys. Fluids 25, 502 (1982).

${ }^{2}$ W. W. Lee, Phys. Fluids 26, 556 (1983).

${ }^{3}$ D. H. E. Dubin, J. A. Krommes, C. Oberman, and W. W. Lee, Phys. Fluids 26, 3524 (1983).

${ }^{4}$ T. S. Hahm, Phys. Fluids 31, 2670 (1988).

${ }^{5}$ T. S. Hahm, W. W. Lee, and A. Brizard, Phys. Fluids 31, 1940 (1988).

${ }^{6}$ A. Brizard, J. Plasma Phys. 41, 541 (1989).

${ }^{7}$ A. Brizard and T. S. Hahm, Rev. Mod. Phys. 79, 421 (2007).

${ }^{8}$ E. Mazzucato and R. Nazikian, Phys. Rev. Lett. 71, 1840 (1993).

${ }^{9}$ F. Wagner, G. Becker, K. Behringer, D. Campbell, A. Eberhagen, W. Engelhardt, G. Fussmann, O. Gehre, J. Gernhardt, G. v. Gierke, G. Haas, M. Huang, F. Karger, M. Keilhacker, O. Klüber, M. Kornherr, K. Lackner, G. Lisitano, G. G. Lister, H. M. Mayer, D. Meisel, E. R. Müller, H. Murmann, H. Niedermeyer, W. Poschenrieder, H. Rapp, H. Röhr, F. Schneider, G. Siller, E. Speth, A. Stäbler, K. H. Steuer, G. Venus, O. Vollmer, and Z. Yü, Phys. Rev. Lett. 49, 1408 (1982).

${ }^{10}$ K. H. Burrell, Phys. Plasmas 4, 1499 (1997).

${ }^{11}$ E. Mazzucato, S. H. Batha, M. Beer, M. Bell, R. E. Bell, R. V. Budny, C. Bush, T. S. Hahm, G. W. Hammett, F. M. Levinton, R. Nazikian, H. Park, G. Rewoldt, G. L. Schmidt, E. J. Synakowski, W. M. Tang, G. Taylor, and M. C. Zarnstorff, Phys. Rev. Lett. 77, 3145 (1996).

${ }^{12}$ E. J. Synakowski, S. Batha, M. Beer, M. G. Bell, R. E. Bell, R. V. Budny, C. E. Bush, P. C. Efthimion, T. S. Hahm, G. Hammett, B. LeBlanc, F. Levinton, E. Mazzucato, H. Park, A. T. Ramsey, G. Schmidt, G. Rewoldt, S. D. Scott, G. Taylor, and M. C. Zarnstorff, Phys. Plasmas 4, 1736 (1997).

${ }^{13}$ R. Nazikian, K. Shinohara, G. J. Kramer, E. Valeo, K. Hill, T. S. Hahm, G. Rewoldt, S. Ide, Y. Koide, Y. Oyama, H. Shirai, and W. Tang, Phys. Rev. Lett. 94, 135002 (2005).

${ }^{14}$ B. D. Scott, Plasma Phys. Controlled Fusion 39, 1635 (1997).

${ }^{15}$ X. Q. Xu, R. H. Cohen, T. D. Rognlien, and J. R. Myra, Phys. Plasmas 7, 1951 (2000)

${ }^{16}$ K. Hallatschek and D. Biskamp, Phys. Rev. Lett. 86, 1223 (2001).

${ }^{17}$ R. J. Fonck, G. Cosby, R. D. Durst, S. F. Paul, N. Bretz, S. Scott, E. Synakowski, and G. Taylor, Phys. Rev. Lett. 70, 3736 (1993).

${ }^{18}$ G. R. McKee, R. J. Fonck, M. Jakubowski, K. H. Burrell, K. Hallatschek,
R. A. Moyer, and D. L. Rudakov, Phys. Plasmas 10, 1712 (2003).

${ }^{19}$ S. Zweben and S. S. Medley, Phys. Fluids B 1, 2058 (1989).

${ }^{20}$ Ch. P. Ritz, R. V. Bravenec, P. M. Schoch, R. D. Bengston, J. A. Boedo, J. C. Forster, K. W. Gentle, Y. He, R. L. Hickok, Y. J. Kim, H. Lin, P. E. Phillips, T. L. Rhodes, W. L. Rowan, P. M. Valanju, and A. J. Wootton, Phys. Rev. Lett. 62, 1844 (1989).

${ }^{21}$ M. Endler, H. Niedermeyer, L. Giannone, E. Kolzhauer, A. Rudyj, G. Theimer, and N. Tsois, Nucl. Fusion 35, 1307 (1995).

${ }^{22}$ J. A. Boedo, D. L. Rudakov, R. A. Moyer, G. R. McKee, R. J. Colchin, M. J. Schaffer, P. G. Stangeby, W. P. West, S. L. Allen, T. E. Evans, R. J. Fonck, E. M. Hollmann, S. Krasheninnikov, A. W. Leonard, W. Nevins, M. A. Mahdavi, G. D. Porter, G. R. Tynan, D. G. Whyte, and X. Xu, Phys. Plasmas 10, 1670 (2003).

${ }^{23}$ K. H. Burrell, E. J. Doyle, P. Gohil, R. J. Groebner, J. Kim, R. J. La Haye, L. L. Lao, R. A. Moyer, T. H. Osborne, W. A. Peebles, C. L. Rettig, T. H. Rhodes, and D. M. Thomas, Phys. Plasmas 1, 1536 (1994).

${ }^{24}$ T. S. Hahm, Phys. Plasmas 3, 4658 (1996).

${ }^{25}$ B. D. Scott, Plasma Phys. Controlled Fusion 45, A385 (2003).

${ }^{26}$ H. Biglari, P. H. Diamond, and P. W. Terry, Phys. Fluids B 2, 1 (1990).

${ }^{27}$ P. H. Diamond, Y.-M. Liang, B. A. Carreras, and P. W. Terry, Phys. Rev. Lett. 72, 2565 (1994).

${ }^{28}$ T. S. Hahm, Phys. Plasmas 1, 2940 (1994).

${ }^{29}$ T. S. Hahm and K. H. Burrell, Phys. Plasmas 2, 1648 (1995).

${ }^{30}$ P. H. Diamond, V. B. Lebedev, D. E. Newman, B. A. Carreras, T. S. Hahm, W. M. Tang, G. Rewoldt, and K. Avinash, Phys. Rev. Lett. 78, 1472 (1996).

${ }^{31}$ T. S. Hahm, Plasma Phys. Controlled Fusion 44, A87 (2002).

${ }^{32}$ P. H. Diamond, S. I. Itoh, K. Itoh, and T. S. Hahm, Plasma Phys. Controlled Fusion 47, R35 (2006).

${ }^{33}$ H. Qin, R. H. Cohen, W. M. Nevins, and X. Q. Xu, Contrib. Plasma Phys. 46, 477 (2006).

${ }^{34}$ H. Qin, R. H. Cohen, W. M. Nevins, and X. Q. Xu, Phys. Plasmas 14, 056110 (2007).

${ }^{35}$ G. Kawamura and A. Fukuyama, Phys. Plasmas 15, 042304 (2008).

${ }^{36}$ R. G. Littlejohn, Phys. Fluids 24, 1730 (1981).

${ }^{37}$ R. G. Littlejohn, J. Math. Phys. 23, 742 (1982).

${ }^{38}$ R. G. Littlejohn, J. Plasma Phys. 29, 111 (1983).

${ }^{39}$ W. M. Tang, Phys. Plasmas 9, 1856 (2002).

${ }^{40}$ A. N. Simakov and P. J. Catto, Phys. Plasmas 12, 012105 (2005).

${ }^{41}$ G. Kagan and P. J. Catto, Plasma Phys. Controlled Fusion 50, 085010 (2008).

${ }^{42}$ Z. Lin, W. M. Tang, and W. W. Lee, Phys. Rev. Lett. 78, 456 (1997).

${ }^{43}$ W. X. Wang, G. Rewoldt, W. M. Tang, F. L. Hinton, J. Manickam, L. E. Zakharov, R. B. White, and S. Kaye, Phys. Plasmas 13, 082501 (2006).

${ }^{44}$ C. S. Chang and S. Ku, Phys. Plasmas 15, 062510 (2008).

${ }^{45}$ X. Q. Xu, Phys. Rev. E 78, 016406 (2008).

${ }^{46}$ T. S. Hahm, Phys. Fluids B 4, 2801 (1992).

${ }^{47}$ M. Artun and W. M. Tang, Phys. Plasmas 1, 2682 (1994).

${ }^{48}$ A. Brizard, Phys. Plasmas 2, 459 (1995).

${ }^{49}$ H. Sugama and W. Horton, Phys. Plasmas 5, 2560 (1998)

${ }^{50}$ R. J. Groebner, K. H. Burrell, and R. P. Seraydarian, Phys. Rev. Lett. 64, 3015 (1990).

${ }^{51}$ R. A. Moyer, K. H. Burrell, T. N. Carlstrom, S. Coda, R. W. Conn, E. J. Doyle, P. Gohil, R. J. Groebner, J. Kim, R. Lehmer, W. A. Peebles, M. Porkolab, C. L. Rettig, T. L. Rhodes, R. P. Seraydarian, R. Stockdale, D. M. Thomas, G. R. Tynan, and J. G. Watkins, Phys. Plasmas 2, 2397 (1995).

${ }^{52}$ M. Ono, S. M. Kaye, Y.-K. M. Peng, G. Barnes, W. Blanchard, M. D. Cartera, J. Chrzanowski, L. Dudek, R. Ewigb, D. Gates, R. E. Hatcher, T. Jarboeb, S. C. Jardin, D. Johnson, R. Kaita, M. Kalish, C. E. Kessel, H. W. Kugel, R. Maingia, R. Majeski, J. Manickam, B. McCormack, J. Menard, D. Mueller, B. A. Nelsonb, B. E. Nelsona, C. Neumeyer, G. Oliaro, F. Paolettic, R. Parsells, E. Perry, N. Pomphrey, S. Ramakrishnan, R. Ramanb, G. Rewoldt, J. Robinson, A. L. Roquemore, P. Ryana, S. Sabbagh, D. Swaina, E. J. Synakowski, M. Viola, M. Williams, J. R. Wilson, and NSTX Team, Nucl. Fusion 40, 557 (2000).

${ }^{53}$ A. Banos, J. Plasma Phys. 1, 305 (1967).

${ }^{54}$ H. L. Berk and A. A. Galeev, Phys. Fluids 10, 441 (1967).

${ }^{55}$ H. P. Furth and M. N. Rosenbluth, in Proceedings of the 3rd International Conference on Plasma Physics and Controlled Nuclear Fusion Research, 1968 (IAEA, Vienna, 1969), Vol. 1, p. 821.

${ }^{56}$ R. D. Hazeltine, Phys. Fluids B 1, 2031 (1989).

${ }^{57}$ T. S. Hahm, Phys. Fluids B 4, 4046 (1992). 
${ }^{58}$ F. L. Hinton and Y. B. Kim, Phys. Plasmas 2, 159 (1995).

${ }^{59}$ K. H. Burrell, Plasma Phys. Controlled Fusion 40, 1585 (1998).

${ }^{60}$ R. Sabot, F. Clairet, G. D. Conway, L. Cupido, X. Garbet, G. Falchetto, T. Gerbaud, S. Hacquin, P. Hennequin, S. Heuraux, C. Honoré, G. Leclert, L. Meneses, A. Sirinelli, L. Vermare, and A. Truc, Plasma Phys. Controlled Fusion 48, B421 (2006)

${ }^{61}$ S. J. Zweben, D. P. Stotler, J. L. Terry, B. LaBombard, M. Greenwald, M. Muterspaugh, C. S. Pitcher, and K. Hallatschek, Phys. Plasmas 9, 1981 (2002).

${ }^{62}$ B. B. Kadomtsev, Plasma Turbulence (Academic, New York, 1965).

${ }^{63}$ W. Horton, Rev. Mod. Phys. 71, 735 (1999).

${ }^{64}$ P. Hennequin, R. Sabot, C. Honoré, G. T. Hoang, X. Garbet, A. Truc, C. Fenzi, and A. Quéméneur, Plasma Phys. Controlled Fusion 46, B121 (2004).

${ }^{65}$ D. Strintzi and B. D. Scott, Phys. Plasmas 11, 5452 (2004).

${ }^{66}$ A. M. Dimits, L. L. LoDestro, and D. H. E. Dubin, Phys. Fluids B 4, 274 (1992).

${ }^{67}$ R. D. Sydora, T. S. Hahm, W. W. Lee, and J. M. Dawson, Phys. Rev. Lett. 64, 2015 (1990).

${ }^{68}$ S. E. Parker, W. W. Lee, and R. A. Santoro, Phys. Rev. Lett. 71, 2042 (1993).

${ }^{69}$ A. M. Dimits, J. A. Byers, T. J. Williams, and B. I. Cohen, Phys. Rev. Lett. 77, 71 (1996).

${ }^{70}$ Z. Lin, T. S. Hahm, W. W. Lee, W. M. Tang, and R. B. White, Science 281, 1835 (1998).

${ }^{71}$ F. Jenko and B. D. Scott, Phys. Plasmas 6, 2705 (1999).

${ }^{72}$ J. Candy, Phys. Plasmas 12, 072307 (2005).

${ }^{73}$ H. Sugama, Phys. Plasmas 7, 466 (2000).

${ }^{74}$ R. E. Waltz, Phys. Fluids 28, 577 (1985).

${ }^{75}$ P. W. Terry, P. H. Diamond, and T. S. Hahm, Phys. Rev. Lett. 57, 1899 (1986).

${ }^{76}$ B. D. Scott, Plasma Phys. Controlled Fusion 39, 1635 (1997).

${ }^{77}$ B. D. Scott, Phys. Plasmas 10, 963 (2003).

${ }^{78}$ M. A. Beer and G. W. Hammett, Phys. Plasmas 3, 4046 (1996).
${ }^{79}$ T. S. Hahm, P. H. Diamond, O. D. Gurcan, and G. Rewoldt, Phys. Plasmas 14, 072302 (2007).

${ }^{80}$ T. S. Hahm, P. H. Diamond, O. D. Gurcan, and G. Rewoldt, Phys. Plasmas 15, 055902 (2008).

${ }^{81}$ A. J. Brizard, Phys. Plasmas 7, 4816 (2000).

${ }^{82}$ A. Kaufman and P. Rostler, Phys. Fluids 14, 446 (1971).

${ }^{83}$ A. Mishchenko, R. Hatzky, and A. Könies, Phys. Plasmas 15, 112106 (2008).

${ }^{84}$ R. M. Kulsrud, in Basic Plasma Physics, edited by A. A. Galeev and R. N. Sudan (North-Holland, Amsterdam, 1983), Vol. I, pp. 115-146.

${ }^{85}$ F. Y. Gang and P. H. Diamond, Phys. Fluids B 2, 2976 (1990).

${ }^{86}$ B. H. Fong and T. S. Hahm, Phys. Plasmas 6, 188 (1999).

${ }^{87}$ Liu Chen, R. L. Berger, J. G. Lominadze, M. N. Rosenbluth, and P. H. Rutherford, Phys. Rev. Lett. 39, 754 (1977).

${ }^{88}$ F. Y. Gang, P. H. Diamond, and M. N. Rosenbluth, Phys. Fluids B 3, 68 (1991).

${ }^{89}$ T. S. Hahm and W. M. Tang, Phys. Fluids B 3, 989 (1991).

${ }^{90}$ J. Lang, Y. Chen, and S. E. Parker, Phys. Plasmas 14, 082315 (2007).

${ }^{91}$ Z. Lin and T. S. Hahm, Phys. Plasmas 11, 1099 (2004).

${ }^{92}$ L. Villard, S. J. Allfrey, and A. Bottino, Nucl. Fusion 44, 172 (2004).

${ }^{93}$ W. X. Wang, T. S. Hahm, W. W. Lee, G. Rewoldt, J. Manickam, and W. M. Tang, Phys. Plasmas 14, 072306 (2007).

${ }^{94}$ W. W. Lee, S. Ethier, R. Kolesnikov, W. X. Wang, and W. M. Tang, Computational Science \& Discovery 1, 015010 (2008).

${ }^{95}$ C. S. Chang, S. Ku, P. H. Diamond, Z. Lin, S. Parker, T. S. Hahm, and N. Samatova, "Compressed ITG turbulence in an L-mode density pedestal in diverted tokamak geometry," Phys. Plasmas (submitted).

${ }^{96}$ P. Sosenko, P. Bertrand, and V. Decyk, Phys. Scr. 64, 264 (2001).

${ }^{97}$ M. Yagi, S. I. Itoh, K. Itoh, A. Fukuyama, and M. Azumi, Phys. Plasmas 2, 4140 (1995).

${ }^{98}$ W. Horton, P. Zhu, G. T. Hoang, T. Aniel, M. Ottaviani, and X. Garbet, Phys. Plasmas 7, 1494 (2000).

${ }^{99}$ K. L. Wong, N. L. Bretz, T. S. Hahm, and E. Synakowski, Phys. Lett. A 236, 339 (1997). 